Fazit: Bei Kindern mit Rhinitis und/oder mildem bis moderatem allergischen Asthma kann eine SLIT die Asthmasymptome lindern. Dieser Effekt bleibt auch 4 bis 5 Jahre nach Absetzen der Behandlung bestehen. $b k$
Di Rienzo V et al. Long-lasting effect of sublingual immunotherapy in children with asthma due to house dust mite: a 10-year prospective study. Clin Exp Allergy 2003; 33: 206-10

\title{
Wirksame Hyposensibilisierung gegen Ameisengift
}

\author{
Bisherige Versuche einer Hyposensibilisierung bei Ameisengift- \\ allergie setzten auf die Applikation von Extrakten ganzer Tiere und \\ verliefen je nach Spezies unterschiedlich erfolgreich. Eine doppel- \\ blinde, plazebokontrollierte Studie untersuchte jetzt Wirksamkeit \\ und Verträglichkeit einer spezifischen Gift-Immuntherapie.
}

A ustralische Allergologen rekrutier65 Jahren mit einer Allergie gegen Myrmecia pilosula. Diese aggressive, Stachel tragende Springameise ist für rund $90 \%$ aller Ameisengiftallergien in Australien verantwortlich. Die Patienten erhielten entweder eine Gift-Immuntherapie (VIT) oder Plazebo, primärer Endpunkt der Studie war die Stichprovokation. Das Insektengift wurde durch Präparation der Giftsäcke von frei lebenden, tasmanischen Springameisen gewonnen. Die geplante Erhaltungsdosis von $100 \mu \mathrm{g}$ sollte nach neun Injektionen erreicht werden.

Zur Auswertung kamen die Daten von 23 VIT- und 29 Plazebopatienten. Nach Stichprovokation zeigten $21 \mathrm{der}$ Patienten unter Plazebo eine systemische Reaktion, dagegen kein Patient aus der Verumgruppe $(p<0,0001)$. Bei zwölf weiteren VIT-Patienten, die schon vor der Stichprovokation bemerkt hatten, dass sie mit Verum behandelt wurden, reagierte ein Patient auf die Stichprovokation mit einer leichten, aber nicht behandlungsbedürftigen Urtikaria. Nach der Behand- lung auch der Plazebopatienten mit VIT zeigte sich hier lediglich bei einem der 26 Patienten noch eine leichte Urtikaria nach Stichprovokation. Systemische Reaktionen als Systemische Reaktionen als
Nebenwirkung wurden bei insgesamt 22 der 64 mit VIT behandelten Patienten gesehen (34\%). Bei zwei Probanden kam es zu ernsthaften Zwischenfällen mit Hypotension und anschließender Bradykardie. Beide erholten sich nach Gabe von Adrenalin, Atropin und Kochsalzinfusionen.

Fazit: Die Hyposensibilisierung mit Ameisengift von Myrmecia pilosula ist gut wirksam. Allerdings muss mit systemischen Reaktionen als Nebenwirkung gerechnet werden, so dass eine Behandlung immer in Zentren mit einer entsprechenden Notfallausrüstung erfolgen sollte.

$b k$

Brown SGA et al. Ant venom immunotherapy: a double-blind, placebo-controlled, crossover trial. Lancet 2003; 361: 1001-6 